\title{
Analysis of A549 cell proteome alteration in response to recombinant influenza A virus nucleoprotein and its interaction with cellular proteins, a preliminary study
}

\author{
D. KUMAR, K. TIWARI, M. S. RAJALA*
}

School of Biotechnology, Jawaharlal Nehru University, New Delhi, India

Received April 19, 2016; revised July 29, 2016; accepted January 18, 2017

\begin{abstract}
Summary. - Influenza A virus undergoes frequent changes of antigenicity and contributes to seasonal epidemics or unpredictable pandemics. Nucleoprotein, encoded by gene segment 5 , is an internal protein of the virus and is conserved among strains of different host origins. In the current study, we analyzed the differentially expressed proteins in A549 cells transiently transfected with the recombinant nucleoprotein of influenza A virus by $2 \mathrm{D}$ gel electrophoresis. The resolved protein spots on gel were identified by MALDI-TOF/Mass spectrometry analysis. The majority of the host proteins detected to be differentially abundant in recombinant nucleoprotein-expressing cells as compared to vector-transfected cells are the proteins of metabolic pathways, glycolytic enzymes, molecular chaperones and cytoskeletal proteins. We further demonstrated the interaction of virus nucleoprotein with some of the identified host cellular proteins. In vitro binding assay carried out using the purified recombinant nucleoprotein (pET29a+NP-His) and A549 cell lysate confirmed the interaction between nucleoprotein and host proteins, such as alpha enolase 1, pyruvate kinase and $\beta$-actin. The preliminary data of our study provides the information on virus nucleoprotein interaction with proteins involved in glycolysis. However, studies are ongoing to understand the significance of these interactions in modulating the host factors during virus replication.
\end{abstract}

Keywords: influenza A virus; A549 cells; nucleoprotein; alpha enolase 1; pyruvate kinase

\section{Introduction}

Influenza A virus, a member of Orthomyxoviridae family, exhibits many well-exemplified mechanisms to evade the innate and adaptive immune responses (Fernandez-Sesma et al., 2006; van de Sandt et al., 2012). It is a highly mutable virus and changes its antigenic properties frequently, contributing to seasonal epidemics and unpredictable pandemics. Incorporation of point mutations in the viral genome by error-prone RNA-dependent RNA polymerase, especially

Corresponding author. E-mail: msrajala@mail.jnu.ac.in; phone +91-11-2673 8752.

Abbreviations: MALDI-TOF = matrix-assisted laser desorption ionization-time of flight; $\mathrm{MS}$ = mass spectrometry; $\mathrm{NP}$ = nucleoprotein in genes encoding surface glycoproteins, hemagglutinin (HA) and neuraminidase (NA), and the reassortment of genomic RNA segments of strains originating from different hosts lead to the emergence of a novel strain (Desselberger et al., 1978). Therefore, the available vaccines and drugs fail to provide the immunity at a global scale. Newly emerged influenza virus strains account for significant mortality and morbidity worldwide, making it a public health threat. Encountering another influenza pandemic is inevitable due to its segmented genetic makeup; however, it is hard to predict the time, host origin and the severity of infection. These uncertainties prompt for the identification of new molecular targets both of virus-encoded or cellular factors to develop effective anti-influenza drugs active across species. Influenza A viral genome comprises eight negative sense RNA segments that together encode for 17 proteins, including the recently discovered 7 proteins (Reviewed by Vasin et al., 
2014). Nucleoprotein (NP), encoded by RNA segment 5 , is a $56 \mathrm{kDa}$ multifunctional protein, whose primary function is to encapsidate the segmented viral genome. Together with the viral RNA and the three RNA polymerase subunits (PA, $\mathrm{PB} 1$ and PB2), NP forms the viral ribonucleoprotein complexes (vRNPs) (Pons et al., 1969). NP shuttles between the nucleus and the cytoplasm, interacting during virus replication with a large number of cellular proteins (Portela et al., 2002) to regulate multiple functions. It facilitates viral RNA transcription, replication and genome packaging.

Currently available antiviral drugs, including drugs under development, are designed largely to target the viral surface proteins, neuraminidase (NA) and ion channel (M2) (Davies et al., 1964; Moscona, 2005; Samson et al., 2013). Two major classes of FDA-approved anti-influenza viral drugs are adamantine-based ion channel (M2) blockers (Amantadine and Rimantadine) and NA inhibitors (Oseltamivir, Zanamivir, and Peramivir). However, none of the inhibitors was shown to provide complete protection against the newly emerged viral strains. Viral envelope proteins, HA and NA, are under pressure of changing to escape the host immune response as they are the first proteins exposed to the neutralizing antibodies. In addition, virus develops various strategies to overcome the therapeutic interventions to establish a successful infection in the host. On the contrary, NP, one of the internal proteins of influenza virus, is highly conserved across the strains originating from different hosts (Shu et al., 1993; Morens et al., 2013). It plays a critical role in genome stabilization as well. A recent study by Kukol and Hughes reported a high degree of conservation among NP sequences using more than 4,400 sequences on host-based characterization (Kukol and Hughes, 2014). Due to conserved amino acid sequences of NP among different strains, it can be a potential target to develop cross-strain protective anti-influenza viral drugs.

In the current study, we sought to analyze the differential expression of host proteins induced by influenza A viral NP. A549 cells were transiently transfected with the recombinant influenza A virus NP (pcDNA3.1 + NP) or empty vector. Protein lysates recovered from transfected cells were subjected to $2 \mathrm{D}$ gel electrophoresis. The resolved proteins on gels were excised and identified by matrix-assisted laser desorption ionization-time of flight/Mass Spectrometry (MALDI-TOF/ MS) analysis. Molecular chaperones, proteins involved in metabolic pathways, glycolytic enzymes and cytoskeletal elements were identified to be differentially expressed in response to recombinant viral NP. Preliminary experiments were carried out to investigate the interaction of NP with few identified host proteins by in vitro binding assay. For the host and viral protein interaction studies, recombinant NP was generated in pET29a+ vector, expressed and purified. Our experiments demonstrated the interaction of NP with host proteins alpha enolase 1 , pyruvate kinase and $\beta$-actin.
All the three proteins were reported to be incorporated into mature influenza virions (Shaw et al., 2010). Nonetheless, it is the first evidence that influenza A virus NP interacts with glycolytic enzymes alpha enolase and pyruvate kinase.

\section{Materials and Methods}

Cells and virus. A549 cell line was obtained from National Centre for Cell Science, Pune, India for propagation of influenza A virus. The cell line was maintained in Ham's Nutrient Mixture F12 supplemented with $10 \%$ FBS and Penicillin-Streptomycin solution. Cells were maintained at $37^{\circ} \mathrm{C}$ in a $5 \% \mathrm{CO}_{2}$ incubator. Influenza A virus, $\mathrm{H} 1 \mathrm{~N} 1$ seasonal strain isolated from a clinical sample was propagated in A549 cells in the laboratory and used in this study.

Generation of recombinant viral nucleoprotein. Total RNA was isolated from virus-infected A549 cells and a fraction of RNA was reverse transcribed using MMULV reverse transcriptase and random hexamer (pdn6). Complementary DNA was amplified using NP gene-specific primers (FP: 5'-ATATGAATTCAC CTGGCGTCCCAAGGCAC-3', RP: 5'-ATATCTCGAGTTAATTG TCGTACTCCTCTGC-3'). Amplified product was purified and cloned into eukaryotic expression vector pCDNA3.1+ driven by CMV promoter between EcoRI and XhoI restriction sites. For in vitro binding studies, NP-encoding viral gene was cloned into bacterial expression vector pET29a+ between the same restriction sites. However, the stop codon was removed from the reverse primer to generate the amplicon to be cloned into $\mathrm{pET}$ vector. Recombinant $\mathrm{NP}$ constructs pCDNA3.1+NP and pET29a+NP-His were confirmed by sequencing.

Transfection and protein recovery. A549 cells were transiently transfected with the recombinant viral NP and mock-transfected with the empty vector pCDNA3.1+ with Lipofectamine LTX reagent (Invitrogen, Life sciences) using the standard protocol. For proteomic analysis, at $48 \mathrm{~h}$ post transfection, cells were washed with $1 \mathrm{xTBS}$ (pH 7.6) and incubated on ice by adding $100 \mu \mathrm{l}$ of 2D extraction buffer (7 mol/l Urea, $2 \mathrm{~mol} / \mathrm{l}$ Thiourea, 2\% CHAPS, $20 \mathrm{mmol} / \mathrm{l}$ Tris-HCl (pH 7.6), 5 mmol/l DTT, $1 \mathrm{mmol} / \mathrm{l} \mathrm{PMSF}$, $1 \mathrm{mmol} / \mathrm{l}$ Sodium orthovanadate, 20 units $/ \mathrm{ml}$ DNases, $0.25 \mathrm{mg} / \mathrm{ml}$ RNases, $0.2 \%$ Bio-Lyte ${ }^{\otimes} 3 / 10$ and $1 x$ Protease inhibitor cocktail) for $30 \mathrm{~min}$. Whole cell lysates were collected and protein concentration was determined.

2D gel electrophoresis. Protein lysate of $169 \mu \mathrm{g}$ was mixed with rehydration buffer ( $8 \mathrm{~mol} / \mathrm{l}$ urea, $2 \%$ CHAPS, $50 \mathrm{mmol} / \mathrm{l}$ DTT, $0.2 \%$ Bio-Lyte $3 / 10$ ampholyte, $0.001 \%$ Bromophenol Blue) to a final volume of $200 \mu$ l. Lysates were loaded onto IPG strips of $7 \mathrm{~cm}$ of a linear broad $\mathrm{pH}$ range of $3-10$ and subjected to isoelectric focusing (IEF). Following IEF, IPG strips were reduced with equilibration buffer I (375 mmol/l Tris-HCl (pH 8.8), 6 mol/l urea, 2\% SDS) containing $2 \%$ DTT for 15 min followed by alkylation with equilibration buffer II containing 2.5\% iodoacetamide for $15 \mathrm{~min}$. IPG strips with separated proteins were subjected to second dimension SDS-PAGE and stained with Gel Code safe blue (Thermo Scientific 
Co, USA). Selected spots were excised using 2D gel spot cutter (Bio-Rad, USA) and subjected to tryptic digestion.

MALDI-TOF/MS analysis. Tryptic digested peptides were loaded onto 384 OptiTOF- MALDI plate (Applied Biosystems) by mixing $0.5 \mu \mathrm{l}$ of the sample with $0.5 \mu \mathrm{l}$ of $1 \%$ a-Cyano- 4 -hydroxycinnamic acid (CHA). Spots were air dried and plates were loaded onto proteome analyser SCIEX TOF/TOF 5800 (Applied Biosystems). The peptides identified by MALDI-TOF/MS were further subjected to MS/MS fragmentation. TOF/TOF TM series Explorer TM Software was used for acquisition and processing of MS/MS spectra. The identified peptides were searched against MASCOT database using Protein Pilot software (AB SCIEX) connected to its server.

Expression and purification of the recombinant viral nucleoprotein (pET29a+NP-His). Recombinant plasmid of viral NP with correct DNA sequence was transformed into BL21 (DE3) pLysS cells and spread on LB plates containing $100 \mu \mathrm{g} / \mathrm{ml}$ kanamycin. Plates were incubated overnight at $37^{\circ} \mathrm{C}$. Next day, a single colony was inoculated in $2 \mathrm{ml} \mathrm{LB}$ with antibiotics and incubated overnight at $37^{\circ} \mathrm{C}$. One percent of the primary culture was used to inoculate $10 \mathrm{ml} \mathrm{LB}$ with antibiotic and allowed to grow till the optical density at $600 \mathrm{~nm}$ reached 0.5 to 0.6 . Upon reaching the desired $\mathrm{OD}$, cells harboring the recombinant construct were induced with $1.0 \mathrm{~mol} / \mathrm{l}$ isopropylbeta-D-thiogalactopyranoside (IPTG) at $32^{\circ} \mathrm{C}$. Following overnight induction, cells were harvested by centrifugation at 5,000 rpm for $10 \mathrm{~min}$ at $4^{\circ} \mathrm{C}$. The cell pellet was resuspended in $200 \mu \mathrm{l}$ of lysis buffer ( $1 \mathrm{mg} / \mathrm{ml}$ lysozyme, $2 \mathrm{~mol} / \mathrm{l} \mathrm{DTT}, 1 \mathrm{mmol} / \mathrm{l} \mathrm{PMSF}, 1 \mathrm{U} / \mathrm{ml}$ of DNase prepared in 1xPBS) followed by sonication until a clear and non-viscous solution was obtained. The cell lysate was clarified by centrifugation at $14,000 \mathrm{rpm}$ for $20 \mathrm{~min}$ at $4^{\circ} \mathrm{C}$. The recombinant protein was purified using metal chelate affinity chromatography under native conditions. For purification of the protein, bacterial cell culture harboring recombinant plasmid was scaled up to $100 \mathrm{ml}$ and cell lysate was prepared as described above. Prior to use, Ni-NTA resin was washed thrice with $1 \mathrm{x}$ PBS (pH 7.4). Fifty $\mu \mathrm{l}$ of Ni-NTA resin was incubated with $150 \mu \mathrm{l}$ of cell lysate made up to $500 \mu \mathrm{l}$ with binding buffer $(2 \mathrm{mmol} / \mathrm{l}$ DTT, $1 \mathrm{mmol} / \mathrm{l} \mathrm{PMSF}$, $20 \mathrm{mmol} / \mathrm{l}$ imidazole in $1 \mathrm{x}$ PBS) for $6 \mathrm{~h}$ at $4^{\circ} \mathrm{C}$. Ni-NTA resin with bound recombinant viral NP was washed thrice with binding buffer. Bound protein was eluted with $50 \mu$ of elution buffer $(50 \mathrm{mmol} / \mathrm{l}$ sodium phosphate buffer ( $\mathrm{pH} 8.0$ ), $300 \mathrm{mmol} / \mathrm{l} \mathrm{NaCl}$ and imidazole). Elution was repeated with elution buffer containing increasing concentrations of imidazole $(250 \mathrm{mmol} / \mathrm{l}$ and $400 \mathrm{mmol} / \mathrm{l})$. Eluted protein was stored at $-80^{\circ} \mathrm{C}$ for further use.

In vitro binding assay. To check the interaction of virus NP with some proteins identified by proteomic analysis, in vitro binding assay was carried out using recombinant NP. In brief, BL21 cells were transformed with recombinant NP (pET29a+NP-His) and induced with $1.0 \mathrm{mmol} / \mathrm{l}$ IPTG overnight at $32^{\circ} \mathrm{C}$. Bacterial cell lysates were prepared and incubated with Ni-NTA beads for $6 \mathrm{~h}$ at $4^{\circ} \mathrm{C}$. Ni-NTA beads with bound His-tagged recombinant NP were washed with $1 \mathrm{xPBS}$. Then, the beads were incubated overnight with $1 \mathrm{mg}$ of A549 cell lysate. To confirm the specific interaction between viral NP and host proteins, an unrelated purified bacterial protein of 28 $\mathrm{kDa}$ was used as a nonspecific control. Next day, Ni-NTA beads were washed, the bound protein complexes were eluted and subjected to SDS-PAGE followed by western blot analysis using antibodies to

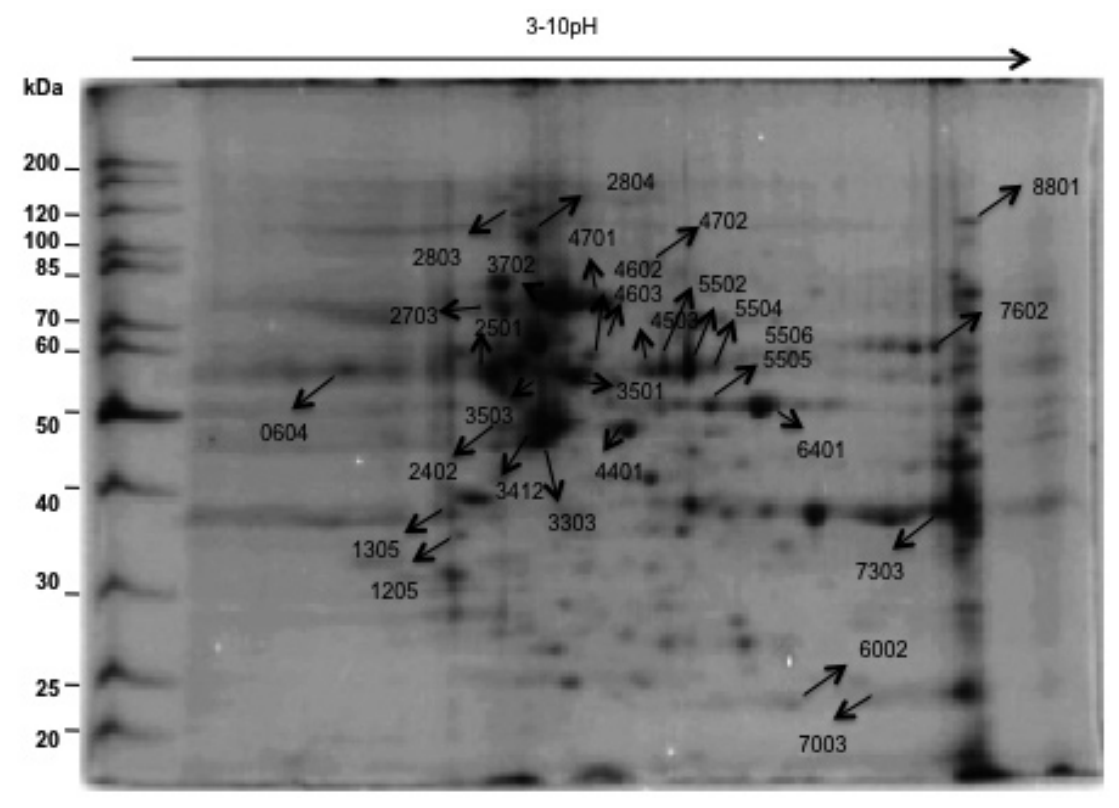

Fig. 1

Representative 2D gel with resolved protein spots of recombinant viral NP-expressing cells

A549 cells were transiently transfected with recombinant viral NP and mock-transfected with empty vector pcDNA3.1(+). At $48 \mathrm{~h}$ post transfection, cell lysates were prepared, subjected to IEF using 3-10 pH range IPG strips followed by 2D gel electrophoresis. Gels were stained with Gel code blue safe. PAGE gel with MS analyzed spots marked and numbered. 


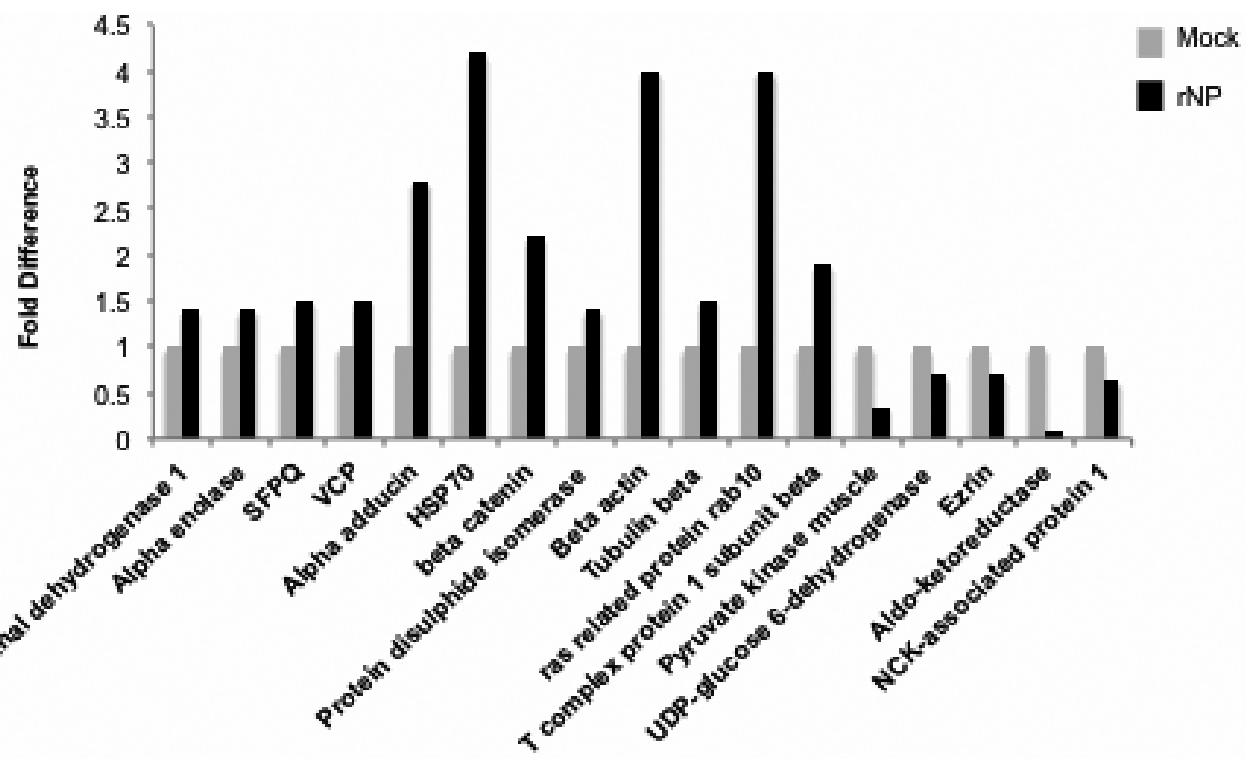

Fig. 2

Graphical representation of MALDI-TOF/MS data showing differential expression of proteins identified in recombinant viral nucleoprotein $v s$. mock transfected cells

alpha enolase 1, pyruvate kinase and $\beta$-actin. BL21 bacterial cells lysate with plain Ni-NTA beads without the recombinant protein was also included as a control.

\section{Results}

\section{Analysis of differentially expressed A549 cell proteome}

Virus NP-encoding gene segment was successfully cloned into eukaryotic expression vector pcDNA3.1 (+) for proteomic analysis and into pET29a+ vector for in vitro binding assay. Recombinant protein expression in A549 cells was confirmed by IFA staining using anti-influenza A virus NP antibody (Merck Millipore, Chemicon). To identify the differentially regulated host proteins induced by influenza A viral NP, cells were transfected with the recombinant NP construct (pCDNA3.1+NP) or mock-transfected with empty vector. Equal amounts of protein lysates recovered from transfected cells were analyzed on $2 \mathrm{D}$ gels. Representative $2 \mathrm{D}$ gel showing resolved protein spots marked is shown (Fig. 1). A total of 32 spots with a significant difference in fold intensity between recombinant protein and mock transfected cell proteome as determined by PD Quest software were selected for analysis. Protein spots were excised from gels and identified by MALDI-TOF/MS analysis. Protein hits were obtained for all the spots. However, only 20 spots had significant Mascot score, the statistical score associated with the match. Expected molecular weight and pI of each protein spot was matched with the obtained results. The fold difference of each spot between mock and recombinant NPexpressing cells was plotted as column graph (Fig. 2). The majority of proteins identified to be differentially abundant are the components of metabolic pathways, glycolysis, molecular chaperones, cytoskeletal remodelling elements and actin filament binding proteins. Mass of each protein detected with Mascot score and the number of peptides matched are shown (Table 1,2). Twelve proteins were found to be up-regulated with significant score. Retinal dehydrogenase, alpha enolase, alpha adducin isoform c, HSP70, TCP1, $\beta$-actin etc., are the proteins up-regulated in response to NP (Table 1); while pyruvate kinase, ezrin, aldoketoreductase, GFAP are out of eight proteins noted to be down-regulated as compared to mock transfected cell proteome (Table 2).

Expression and purification of His-tagged recombinant viral nucleoprotein

To investigate the viral NP interaction with some of the differentially expressed host proteins, recombinant viral protein was purified and in vitro binding assay was carried out using A549 cell lysates. The bacterial BL21 cells containing recombinant viral NP construct (pET29a+ NP-His) were grown and the expression was induced with $1.0 \mathrm{mmol} / \mathrm{l}$ IPTG. The effect of induction temperature on the level of recombinant protein expression was optimized by incubating the cultures at temperature ranging from 28 to $32^{\circ} \mathrm{C}$ and allowed to grow overnight. Of total protein content, 
Table 1. MALDI-TOF/MS data: Up-regulated proteins in A549 cells transfected with recombinant viral nucleoprotein

\begin{tabular}{|c|c|c|c|c|c|c|}
\hline Spot No. & Protein identified & Accession ID & $\begin{array}{l}\text { Molecular } \\
\text { weight }\end{array}$ & pI & Mascot score & $\begin{array}{l}\text { No. of peptides } \\
\text { matched }\end{array}$ \\
\hline 5504 & Retinal dehydrogenase 1 & gi|2136117 & 55454 & 6.3 & 470 & 36 \\
\hline 6401 & Alpha enolase isoform 1 & gi| 4503571 & 47481 & 7.01 & 550 & 38 \\
\hline 8801 & $\begin{array}{l}\text { Splicing factor proline/glutamine-rich } \\
\text { (SFPQ) }\end{array}$ & gi|119627826 & 66420 & 9.45 & 155 & 18 \\
\hline 2804 & $\begin{array}{l}\text { Transitional Endoplasmic Reticulum ATPase/ } \\
\text { valosin containing protein }\end{array}$ & gi|6005942 & 89950 & 5.14 & 429 & 48 \\
\hline 2803 & Alpha adducin isoform c & gi|29826323 & 70397 & 5.6 & 24 & 20 \\
\hline 3702 & HSP70 & gi|62897129 & 70294 & 5.48 & 90 & 16 \\
\hline 2703 & $\begin{array}{l}\text { Unnamed protein product (highly similar to } \\
\text { beta catenin) }\end{array}$ & gi|194389640 & 78097 & 5.53 & 30 & 9 \\
\hline 4602 & $\begin{array}{l}\text { Protein disulphide isomerase family A, mem- } \\
\text { ber } 3 \text { isoform CRA_a }\end{array}$ & gi|119597640 & 54454 & 5.98 & 237 & 19 \\
\hline 3412 & Beta actin variant/ACTB protein & gi|15277503 & 42080 & 5.29 & 222 & 16 \\
\hline 2501 & Tubulin beta & gi|57209813 & 48135 & 4.78 & 308 & 31 \\
\hline 7003 & $\begin{array}{l}\text { Hypothetical protein (ras related protein } \\
\text { rab10) }\end{array}$ & gi|12052826 & 22683 & 8.58 & 22 & 11 \\
\hline 4503 & T complex protein 1 subunit beta & gi|5453603 & 57766 & 6.01 & 156 & 20 \\
\hline
\end{tabular}

high-level expression of the desired protein of $56 \mathrm{kDa}$ was obtained from cells cultured overnight at $32^{\circ} \mathrm{C}$ (Fig. 3a). Cells were disrupted by sonication followed by centrifugation to separate the soluble fraction from the insoluble one. The recombinant protein content was comparatively equal in both the fractions - pellet and supernatant. Localization of the recombinant protein in both fractions was analyzed by SDS-PAGE (Fig. 3b). Small-scale protein purification was carried out with supernatants by affinity chromatography. For protein purification, initially $100 \mathrm{ml}$ culture was taken. Binding of the recombinant protein to Ni-NTA resin was optimized using different concentrations of imidazole (10 mmol/l, $20 \mathrm{mmol} / \mathrm{l}$, and $30 \mathrm{mmol} / \mathrm{l})$. Also, elution of bound protein was optimized with elution buffer containing varying concentration of imidazole ranging from $200 \mathrm{mmol} / \mathrm{l}$ to $400 \mathrm{mmol} / \mathrm{l}$. Optimal yield of $\sim 56 \mathrm{kDa}$ purified NP was obtained with the binding buffer and elution buffer containing $20 \mathrm{mmol} / \mathrm{l}$ and $250 \mathrm{mmol} / \mathrm{l}$ imidazole, respectively (Fig. 3c). Purified NP identity was confirmed by western blot analysis using anti-His (Fig. 3d) and anti-influenza viral NP antibodies (Fig. 3e).

Nucleoprotein interaction with host proteins identified by proteomic analysis

Interestingly, alpha enolase 1 , pyruvate kinase, $\beta$-actin and aldoketoreductase that were identified to be differentially abundant in cells expressing recombinant viral NP in our study had been reported to be incorporated into mature influenza virion by proteomic analysis (Shaw et al., 2010).

Table 2. MALDI-TOF/MS data: Down-regulated proteins in A549 cells expressing recombinant viral nucleoprotein

\begin{tabular}{|c|c|c|c|c|c|c|}
\hline Spot No. & Protein identified & Accession ID & $\begin{array}{l}\text { Molecular } \\
\text { weight }\end{array}$ & pI & Mascot score & $\begin{array}{c}\text { No. of peptides } \\
\text { matched }\end{array}$ \\
\hline 7602 & $\begin{array}{l}\text { Pyruvate Kinase } \\
\text { muscle }\end{array}$ & gi|31416989 & 58512 & 7.96 & 333 & 42 \\
\hline 6604 & UDP-glucose 6-dehydrogenase isoform1 & gi|296040443 & 55674 & 6.73 & 130 & 18 \\
\hline 0604 & Tubulin alpha $1 \mathrm{~b}$ chain isoform 2 & gi| 18204869 & 46797 & 4.94 & 71 & 8 \\
\hline 4702 & Ezrin & gi|28948869 & 69484 & 5.94 & 116 & 29 \\
\hline 4701 & $\begin{array}{l}\text { Unnamed protein product (highly similar to } \\
\text { homo sapiens albumin) }\end{array}$ & gi|158258947 & 71218 & 5.92 & 44 & 12 \\
\hline 7303 & Aldo-ketoreductase family 1 member c3 & gi|24497583 & 37229 & 8.06 & 170 & 23 \\
\hline 4401 & $\begin{array}{l}\text { NCK-associated } \\
\text { protein } 1\end{array}$ & gi|119631362 & 43731 & 6.18 & 26 & 7 \\
\hline 2402 & Glial fibrillary acidic protein & gi|119571952 & 49776 & 5.42 & 39 & 10 \\
\hline
\end{tabular}


(a)

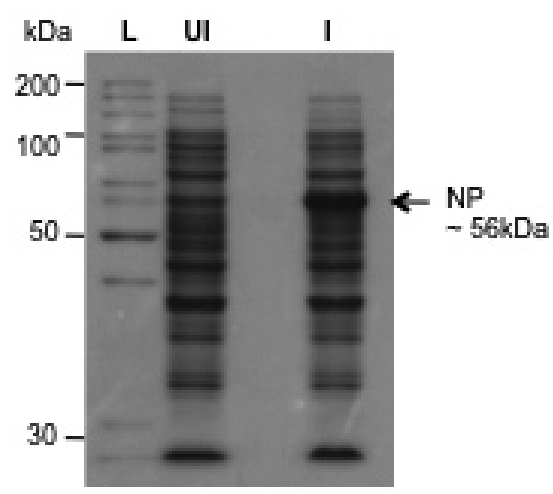

(c)

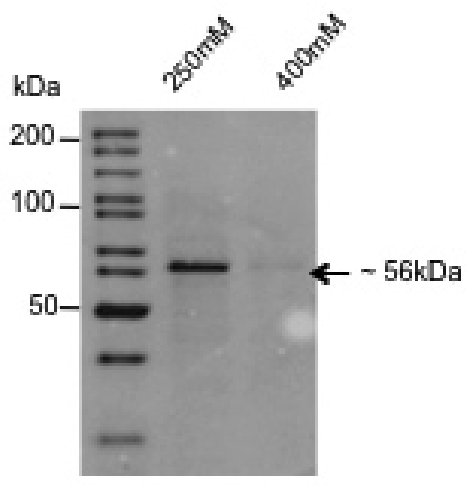

(d) (b)
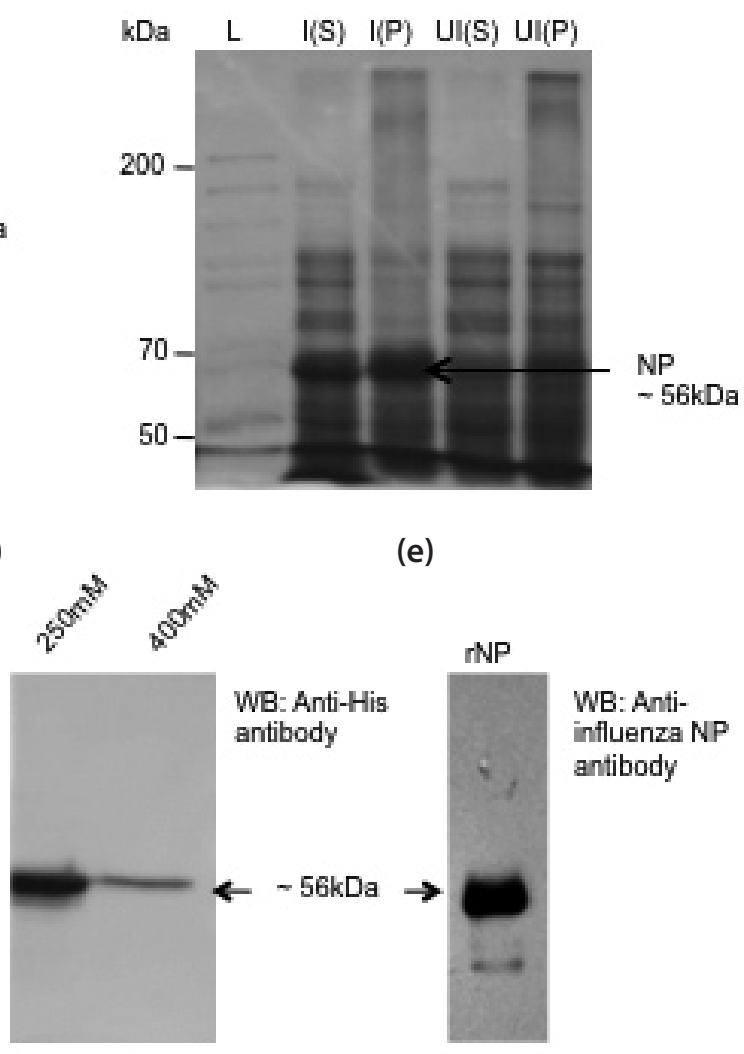

(e)

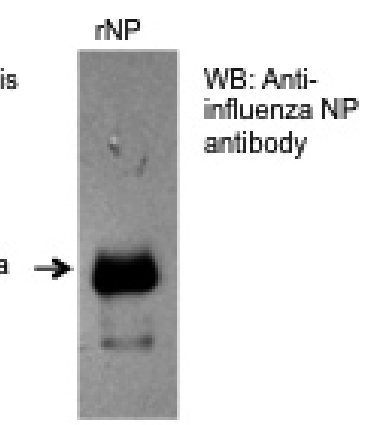

Fig. 3

Recombinant viral nucleoprotein expression and purification

NP-encoding viral gene segment was cloned into a pET29a+ vector. BL21 bacterial cells were transformed with recombinant NP (rNP) of influenza virus; NP was expressed, purified by affinity chromatography and eluted (a) SDS-PAGE analysis of bacterial cell cultures grown in LB medium showing increased expression of the recombinant protein following overnight IPTG induction (I) in comparison with uninduced (UI) culture (b) Localization of recombinant protein; an equal amount of recombinant protein in supernatant and pellet of induced (I(S), I(P)) in comparison with uninduced cultures (UI(S), UI(P)) (c) Eluted recombinant protein resolved on SDS-PAGE using buffer with different concentrations of imidazole (d) Confirmation of recombinant viral $\mathrm{NP}$ of $56 \mathrm{kDa}$ by western blot (WB) analysis using anti-His antibody (e) anti-influenza NP antibody.

However, the mechanism through which the cellular proteins get incorporated in to the mature virion is not clear. Rationally, cellular proteins need to be associated with the viral components at the time of assembly to be packaged into the mature virion. Multiple subunits of NP encapsidate the eight segments of negative sense RNA genome independently to form ribonucleoprotein complexes. Hence, the incorporation of cellular proteins into the mature virion perhaps is through interaction with NP or matrix (M1) protein that forms the inner lining of viral envelope. An attempt was made to investigate the interaction of viral NP with the above identified proteins by in vitro binding assay using recombinant NP (pET29a+NP-His) bound to Ni-NTA resin and A549 cell lysates. Eluted protein complexes from Ni-NTA resin were analyzed by anti-alpha enolase 1 , anti-pyruvate kinase, anti- $\beta$-actin, anti-viral NP and anti-His antibodies. In vitro binding assay confirmed our preliminary hypothesis of interaction of host proteins under test with the recombinant NP (Fig. 4a). However, no such interaction was noted with the unrelated bacterial protein, a nonspecific control used in the assay. Anti-His antibody confirmed the expression of purified recombinant viral NP of $\sim 56 \mathrm{kDa}$ and an unrelated control protein of $\sim 28 \mathrm{kDa}$ in the eluted immune complexes (Fig. 4b). Anti-influenza NP antibody further confirmed the recombinant viral NP expression. Endogenous expression of alpha-enolase 1, pyruvate kinase and $\beta$-actin in A549 cell lysates recovered after the formation of immune complexes was shown by western blot analysis using specific antibodies (Fig. 4c). The significance of host proteins being carried by influenza virus into the new susceptible host and the interaction of NP with alpha enolase and pyruvate kinase is an important aspect to be explored. 
(a)

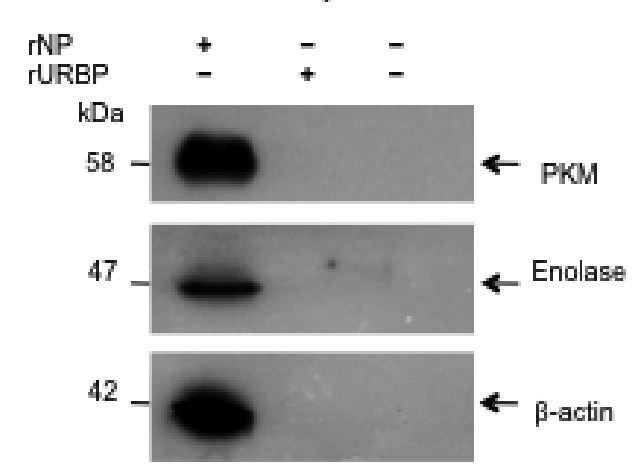

(c)

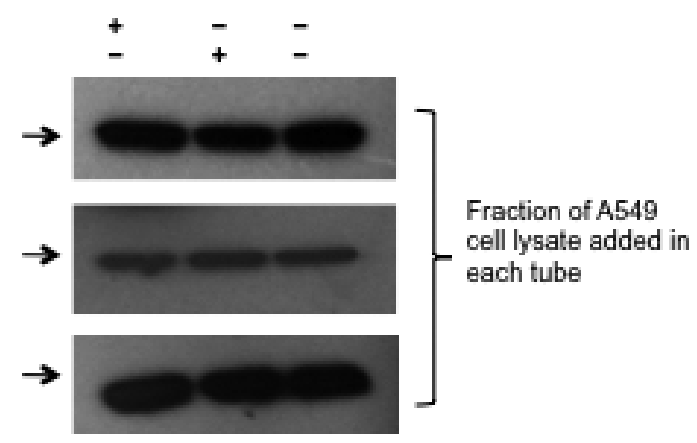

(b)

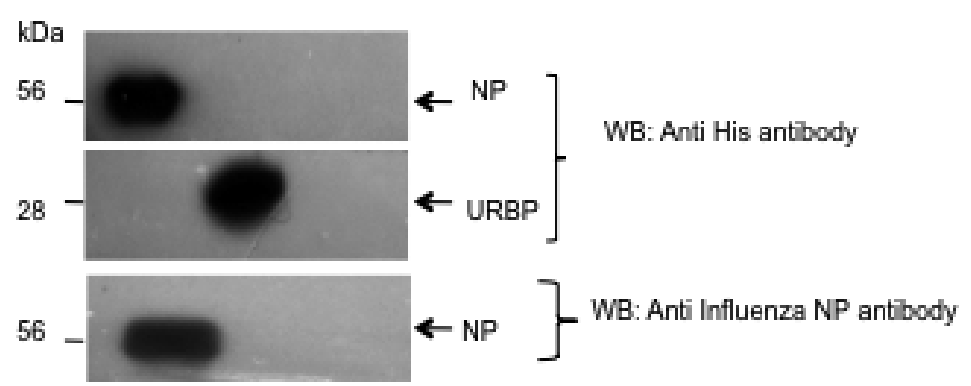

Fig. 4

In vitro binding assay

Cell lysates prepared from BL21 cells expressing recombinant viral NP or unrelated bacterial protein (URBP) with His-tag were incubated with Ni-NTA beads and allowed to immobilize the recombinant protein onto the resin. Beads with bound proteins were washed and further incubated overnight with equal amount of total protein recovered from A549 cells. Next day, protein lysates were centrifuged to pellet down the beads. Protein complexes bound to the beads were eluted and subjected to western blot (WB) analysis using specific antibodies (a) Western blot showing pyruvate kinase, alpha enolase 1 and $\beta$-actin complexed with the recombinant NP of influenza A virus but not with the unrelated bacterial protein. No such band was seen in control; bacterial lysate without any recombinant protein (b) Western blot showing $56 \mathrm{kDa}$ recombinant NP and $28 \mathrm{kDa}$ recombinant non-specific bacterial protein as analyzed by anti-His antibody and anti-influenza NP antibody (c) Confirmation of endogenous levels of pyruvate kinase, alpha enolase and $\beta$-actin in a fraction of total A549 protein lysate recovered from each tube by centrifugation following overnight incubation with recombinant protein bound to Ni-NTA beads (virus NP, URBP, no protein).

\section{Discussion}

In the recent past, internal proteins of influenza $A$ virus such as NP and non-structural protein (NS1) are gaining much importance as an alternate drug targets. NP, due to its conserved protein sequences among strains originating from different hosts was depicted as a potential drug target (Babar et al., 2015). Therefore, the identification of host proteins regulated specifically in response to NP and knowledge on its interaction with host proteins could be an important consideration to design new anti-influenza viral therapeutics. With this point in mind, we evaluated the altered A549 cell proteome induced by influenza A virus recombinant NP by $2 \mathrm{D}$ gel electrophoresis coupled with MALDI-TOF/MS analysis. Considerable number of studies earlier reported the differential regulation of host proteins induced by influenza
A viral strains. Interestingly, the protein profile in our study is strikingly similar to the published influenza A virus-infected cell proteome. Alpha-enolase 1, tubulin $\beta$, protein disulfide isomerase, splicing factor proline/glutamine-rich (SFPQ) proteins had been reported to be regulated in response to H1N1 strain (Zhao et al., 2013). The differential abundance of aldoketoreductase, alpha enolase 1, UDP-glucose-6dehydrogenase in response to H5N1 strain (Liu et al., 2012), T-complex protein 1 in H3N2 infected cells (Wu et al., 2013) and deregulation cell cycle proteins and lipid metabolism by H1N1 2009 pandemic strain (Dove et al., 2012) were demonstrated. These reports collectively suggest the usage of common host factors by influenza A viral strains of different host origins.

The above mentioned studies were carried out using a complete virus to investigate the altered proteome 
of infected cell. Very few studies had demonstrated the impact of a particular viral protein independent of other viral components. Host cellular protein PACT, an essential co-factor for IFN stimulation, had been identified to be an interacting partner to influenza A viral recombinant NS1 protein, a known inhibitor of innate and adaptive immunity by quantitative proteomic analysis. This interaction was shown to block PACT/RIG1-mediated IFN I activation and serve the virus to escape the host immune response (Tawaratsumida et al., 2014). Host response to RNA polymerase complex of $\mathrm{H} 5 \mathrm{~N} 1$ avian strain had also been demonstrated by proteomic analysis and its association with mitochondrial proteins is presumed to be the regulator of apoptosis in host cell (Bradel-Tretheway et al., 2016). Here, we had shown the differential expression of host proteins induced by influenza A viral NP alone.

Generally, enveloped viruses including influenza A virus bud from the host cell plasma membrane and are released into extracellular environment. During this process, some cell surface proteins are packaged into virus particles. Other than influenza A virus, packaging of cellular proteins was accounted for in other viruses, to specify few: human CMV (Varnum et al., 2004), HIV (Cen et al., 2004, Linde et al., 2013), EBV (Johannsen et al., 2004). Host proteins packaged into influenza A virus were grouped into several categories including proteins of metabolic pathways, glycolysis, annexins, and cytoskeletal elements (Shaw et al., 2010). Our preliminary studies demonstrated the interaction of alpha enolase 1 , pyruvate kinase and $\beta$-actin with the recombinant NP (pET29a+NP-His construct) by in vitro binding assay. It proposes the possible role of NP in packaging of the host proteins into mature virus particle; or the described interactions may regulate an alternate function during infection. The association of cytoskeletal elements actin and tubulin with influenza virus matrix protein (M1) and NP has been reported earlier (Avalos et al., 1997), but the interaction of NP with alpha enolase 1 and pyruvate is found to be novel and needs further confirmation.

Alpha enolase is a key glycolytic enzyme expressed in the cytoplasm and on cell surface as well. In addition to its fundamental glycolytic function, it plays a significant role in several biological and pathological processes. So does pyruvate kinase, another key player in glycolysis. Enolase expression was correlated with tumor cell proliferation (Song et al., 2014) and plays a significant role in tumor invasion by modulating oncogenes transcription control (Fu et al., 2015). Similarly, localization of pyruvate kinase, a rate-limiting glycolytic enzyme in the nucleus was correlated with cell proliferation (Gao et al., 2012). Alpha enolase had also been described as a neurotrophic factor (Takei et al., 1991), a heat-shock protein (HSP48) (Iida and Yahara, 1985) and hypoxic stress protein (Aaronson et al., 1995). Likewise, pyruvate kinase has been described as a regula- tor of growth and apoptosis (Spoden et al., 2009) and an immunomodulator in tumor cells (Zhang et al., 2010). Oncogenic viruses are known to control the cell proliferation and DNA damage through modulating glucose metabolic pathways (Noch and Khalili, 2012), but increasing evidence on differential regulation of glycolytic enzymes and other metabolic enzymes in infected cells specify their importance in virus life cycle. Few studies reported the secretion of high level of alpha enolase in dengue-infected hepatic cells (Higa et al., 2014), differential expression of enolase in response to chikungunya virus (Thio et al., 2013) and of pyruvate kinase to bovine herpes simplex 1 virus (Guo et al., 2015). In spite of the fact that the differential expression of glycolytic and other metabolic enzymes in response to viruses including influenza $A$ virus had been accounted for in the literature, neither their interaction with viral proteins nor their functional significance in virus replication is clearly known. One study demonstrated the essential role of enolase and pyruvate kinase in transcription of Sendai virus genome (Ogino et al., 2001).

Based on our preliminary data, we hypothesize that alpha enolase and pyruvate kinase may facilitate the virus assembly and packaging through their interaction with ribonucleoprotein complex. Secondly, when a mature virus infects another susceptible host, the two virion associated proteins, enolase and pyruvate kinase, are released into the host cell and may regulate the initial stages of infection or modulate the host cell glycolysis to create favorable environment for the virus to establish the infection. Likewise, other incorporated proteins may play a regulatory role during infection. Packaging of cellular proteins into virus particles was thought to be accidental, but recent studies demonstrated the vital role of incorporated host proteins for establishing new infection in a susceptible host. Ubiquitin, a regulatory protein incorporated into mature influenza virion was shown to play a crucial role in the internalization of virus during subsequent infection (Banerjee et al., 2014). Similarly, incorporated annexin V counteracts the anti-influenza viral activity by disrupting $\gamma$ IFN signaling and promotes the virus replication in the new host (Berri et al., 2014). Packaging of glycolytic enzymes alpha enolase and pyruvate kinase into mature virion, perhaps through viral NP interaction, is expected to have a significant role in virus infections.

To conclude, incorporation of alpha enolase and pyruvate kinase into mature virion as demonstrated by Shaw et al. (2010), differential expression and interaction with NP as shown by us together indicate their potential role in virus life cycle. Further studies on implications of identified host proteins in response to influenza A virus infection with special reference to the conserved internal NP will possibly help to design and develop novel interventional strategies to combat the infection by different strains of influenza A virus. 
Acknowledgement. This study was supported by Indian Council of Medical Research (No. VIR/15/2011-ECD-I), UPOE II (Project ID-56), and DST-PURSE grant, New Delhi. We acknowledge the Mass spectrometry facility of Advanced Instrumentation Research Facility at JNU, New Delhi, India.

\section{References}

Aaronson RM, Graven KK, Tucci M, McDonald RJ, Farber HW (1995): Non-neuronal enolase is an endothelial hypoxic stress protein. J. Biol. Chem. 270, 27752-27757. https:// doi.org/10.1074/jbc.270.46.27752

Avalos RT, Yu Z, Navak DP (1997): Association of influenza virus $\mathrm{NP}$ and M1 proteins with cellular cytoskeletal elements in influenza virus-infected cells. J. Virol. 71, 2947-2958.

Babar MM, Zaidi NS (2015): Protein sequence conservation and stable molecular evolution reveals influenza A virus nucleoprotein as universal drug target. Infection, Genet. Evolut. 34, 200-210. https://doi.org/10.1016/j. meegid.2015.06.030

Banerjee I, Miyake Y, Nobs SP, Schneider C, Horvath P, Kopf M, Matthias P, Helenius A, Yamauchi Y (2014): Influenza A virus uses the aggresome processing machinery for host cell entry. Science 346, 473-477. https://doi.org/10.1126/ science. 1257037

Berri F, Haffar G, Ba Le V, Sadewasser A, Paki K, Lina B, Wolff T, Riteau B (2014): Annexin V Incorporated into Influenza Virus Particles Inhibits Gamma Interferon Signaling and Promotes Viral Replication. J. Virol. 88, 11215-11228. https://doi.org/10.1128/JVI.01405-14

Bradel-Tretheway BG, Mattiacio JL, Krasnoseksky A, Stevenson C, Purdy D, Dewhurst S, Katze MG (2011): Comprehensive Proteomic Analysis of Influenza Virus Polymerase Complex Reveals a Novel Association with Mitochondrial Proteins and RNA polymerase Accessory Factors. J. Virol. 85, 8569-8581. https://doi.org/10.1128/JVI.00496-11

Cen S, Guo F, Niu M, Saadatmand J, Deflassieux J, Kleiman L (2004): The interaction between HIV-1 Gag and APOBEC3G. J. Biol. Chem. 279, 33177-33184. https://doi.org/10.1074/ jbc.M402062200

Davies WL, Hoffmann CE, Paulshock M, Wood TR, Haff RF, Grunert RR, Watts JC, Hermann EC, Neumayer EM, Mcgahen JW (1964): Antiviral activity of 1-adamantanamine (amantadine). Science 144, 862-863. https://doi. org/10.1126/science.144.3620.862

Desselberger U, Nakajima K, Alfino P, Pedersen FS, Haseltine WA, Hannoun C, Palese P (1978): Biochemical evidence that new influenza virus strains in nature may arise by recombination (reassortment). Proc. Natl. Aca. Sci. USA. 75, 3341-3345. https://doi.org/10.1073/pnas.75.7.3341

Dove BK, Surtees R, Bean TJH, Munday D, Wise HM, Digard P, Carroll MW, Ajuh P, Barr JN, Hiscox JA (2012): A quantitative proteomic analysis of lung epithelial (A549) cells infected with 2009 pandemic influenza A virus using stable isotope labelling with amino acids in cell culture. Proteomics 12, 1431-1436. https://doi.org/10.1002/ pmic. 201100470
Fernandez-Sesma A, Marukian S, Ebersole BJ et al. (2006): Influenza virus evades innate and adaptive immunity via the NS1 protein. J. Virol. 80, 6295-6304. https://doi. org/10.1128/JVI.02381-05

Fu QF, Liu Y, Fan Y, Hua SN, Qu HY et al. (2015): Alpha-enolase promotes cell glycolysis, growth, migration, and invasion in non-small cell lung cancer through FAK-mediated PI3K/AKT pathway. J. Hematol. Oncol. 8, 22. https://doi. org/10.1186/s13045-015-0117-5

Gao X, Wang H, Yang JY, Liu X, Liu ZR (2012): Pyruvate Kinase M2 Regulates Gene Transcription by Acting as a Protein Kinase. Mol. Cell. 45, 598-609. https://doi.org/10.1016/j. molcel.2012.01.001

Guo L, Yang Y, Liu L, Liao P, Wen Y, Wu H, Cheng S (2015): A proteomic study of the differential protein expression in MDBK cells after bovine herpes virus type 1 infection (BHV-1) strain treatment. Int. J. Clin. Exp. Med. 8, 4204-4211.

Higa LM, Curi BM, Aguiar RS, Cardoso CC, De Lorenzi AG, Sena SLF, Zingali RB, Da Poian AT (2014): Modulation of $\alpha$-Enolase Post-Translational Modifications by Dengue Virus: Increased Secretion of the Basic Isoforms in Infected Hepatic Cells. PLoS One 9, e88314. https://doi. org/10.1371/journal.pone.0088314

Iida H, Yahara I (1985): Yeast heat-shock protein of $\mathrm{Mr} 48,000$ is an isoprotein of enolase. Nature 315, 688-690. https:// doi.org/10.1038/315688 $\mathrm{a} 0$

Johannsen E, Luftig M, Chase MR, Weicksel S, Cahir-McFarland E, Illanes D, Sarracino D, Kieff E (2004): Proteins of purified Epstein-Barr virus. Proc. Natl. Acad. Sci. USA 101, 16286-16291. https://doi.org/10.1073/pnas.0407320101

Kukol A, Hughes DJ (2014): Large-scale analysis of influenza virus nucleoprotein sequence conservation reveals potential drug-target sites. Virology 454, 40-47 https://doi. org/10.1016/j.virol.2014.01.023

Linde ME, Colquhoun DR, UbaidaMohien C, Kole T, Aquino V, Cotter R, Edwards N, Hildreth JE, Graham D (2013): The conserved set of host proteins incorporated into HIV-1 virions suggests a common egress pathway in multiple cell types. J. Proteome. Res. 12, 2045-2054. https://doi. org $/ 10.1021 / \mathrm{pr} 300918 \mathrm{r}$

Liu C, Zhang A, Guo J, Yang J, Zhou H, Chen H, Jin M (2012): Identification of human host proteins contributing to H5N1 Influenza virus propagation by membrane proteomics. J. Proteome. Res. 11, 5396-5405. https://doi. org $/ 10.1021 / \mathrm{pr} 3006342$

Morens DM, Taubenberger JK, Fauci AS (2013): Pandemic influenza viruses-hoping for the road not taken. N. Engl. J. Med. 368, 2345-2348. https://doi.org/10.1056/ NEJMp1307009

Moscona A (2005): Neuraminidase inhibitors for influenza. N. Engl. J. Med. 353, 1363-1373. https://doi.org/10.1056/ NEJMra050740

Noch E, Khalili K (2012): Oncogenic Viruses and Tumor Glucose Metabolism: Like Kids in a Candy Store. Mol. Cancer. Ther. 11, 14-23. https://doi.org/10.1158/1535-7163. MCT-11-0517

Ogino T, Yamadera T, Nonaka T, Imajoh-Ohmi S, Mizumoto K (2001): Enolase, a cellular glycolytic enzyme, is required 
for efficient transcription of Sendai virus genome. Biochem. Biophys. Res. Commun. 285, 447-455. https://doi. org/10.1006/bbrc.2001.5160

Pons MW, Schulze IT, Hirst GK, Hauser R (1969): Isolation and characterization of the ribonucleoprotein of influenza virus. Virology 39, 250-259. https://doi.org/10.1016/00426822(69)90045-2

Portela A, Digard P (2002): The influenza virus nucleoprotein: a multifunctional RNA-binding protein pivotal to virus replication. J. Gen. Virol. 83, 723-734. https://doi. org/10.1099/0022-1317-83-4-723

Samson M, Pizzorno A, Abed Y, Boivin G (2013): Influenza virus resistance to neuraminidase inhibitors. Antiviral Res. 98, 174-185. https://doi.org/10.1016/j.antiviral.2013.03.014

Shaw MG, Stone KL, Colangelo CM, Gulcicek EE, Palese P (2010): Cellular proteins in influenza virus particles. PLoS Pathogens 4, e1000085. https://doi.org/10.1371/journal.ppat.1000085

Shu LL, Bean WJ, Webster RG (1993): Analysis of the evolution and variation of the human influenza A virus nucleoprotein gene from 1933 to 1990. J. Virol. 67, 2723-2729.

Song Y, Luo Q, Long H, Hu Z, Que T et al. (2014): Alpha-enolase as a potential cancer prognostic marker promotes cell growth, migration, and invasion in glioma. Molecular Cancer 13, 65. https://doi.org/10.1186/1476-4598-13-65

Spoden GA, Rostek U, Lechner S, Mitterberger M, Mazurek S, Zwerschke W (2009): Pyruvate kinase isoenzyme M2 is a glycolytic sensor differentially regulating cell proliferation, cell size and apoptotic cell death dependent on glucose supply. Exp. Cell. Res. 315, 2765-2774. https:// doi.org/10.1016/i.yexcr.2009.06.024

Takei N, Kondo J, Nagaike K, Ohsawa K, Kato K, Kohsaka (1991): Neuronal survival factor from bovine brain is identical to neuron-specific enolase. J. Neurochem. 57, 1178-1184. https://doi.org/10.1111/j.1471-4159.1991.tb08277.x

Tawaratsumida K, Phan V, Hrincius ER, High AA, Webby R, Redecke V, Hacker H (2015): Quantitative Proteomic Analysis of the Influenza A Virus Nonstructural Proteins NS1 and NS2 during Natural Cell Infection Identifies PACT as an NS1 Target Protein and Antiviral Host Factor. J. Virol. 88, 9038-9048. https://doi.org/10.1128/JVI.00830-14

Thio CLP, Yusof R, Abdul-Rahman PSA, Karsani SA (2013): Differential Proteome Analysis of Chikungunya Virus Infection on Host Cells. PLoS One 8, e61444. https://doi. org/10.1371/journal.pone.0061444

Van de Sandt CE, Kreijtz JHCM, Rimmerlzwaan GF ((2012): Evasion of Influenza A Viruses from Innate and Adaptive Immune Responses. Viruses. 4, 1438-1476. https://doi. org/10.3390/v4091438

Varnum SM, Streblow DN, Monroe ME, Smith P, Auberry KJ, PasaTolic L, Wand D, Camp DG 2nd, Rodland K, Wiley S, Britt W, Shenk T, Smith RD, Nelson JA (2004): Identification of proteins in human cytomegalovirus (HCMV) particles: the HCMV proteome. J. Virol. 78, 10960-10966. https:// doi.org/10.1128/JVI.78.20.10960-10966.2004

Vasin AV, Temkina OA, Egorov VV, Klotchenko SA, Plotnikova MA, Kiselev OI (2014): Molecular mechanisms enhancing the proteome of influenza A viruses: An overview of recently discovered proteins. Virus Res. 185, 53-63. https://doi. org/10.1016/j.virusres.2014.03.015

Wu X, Wang H, Bai L, Yu Y, Sun Z, Zhou J (2013): Mitochondrial proteomic analysis of human host cells infected with H3N2 swine influenza virus. J. Proteomics 91, 136-150. https://doi.org/10.1016/j.jprot.2013.06.037

Zhang Z, Liu Q, Che Y, Yuan X, Dai L, Zeng B, Jiao G, Zhang Y, Wu X, Yu Y, Yang R (2010): Antigen presentation by dendritic cells in tumors is disrupted by altered metabolism that involves pyruvate kinase M2 and its interaction with SOCS3. Cancer Res. 70, 89-98. https://doi. org/10.1158/0008-5472.CAN-09-2970

Zhao H, Yang J, Li K, Ding X, Lin R, Ma Y, Liu J, Zhong Z, Qian X, Bo X, Zhou Z, Wang S (2013): Proteomic analysis at the subcellular level for host targets against influenza A virus (H1N1). Antiviral Res. 100, 673-687. https://doi. org/10.1016/j.antiviral.2013.10.005 